\title{
Prevalence of bilateral 'mirror-image' lesions in patients with oral potentially malignant epithelial lesions
}

Type:

Article

Abstract:

Early detection of oral potentially malignant epithelial lesions (PMELs) is aimed at improving survival rates as carcinogenesis is a multistep process and prevention is possible if these lesions are detected at an early and reversible stage of the disease. A prospective clinical study aimed at determining the prevalence of bilateral 'mirror-image' oral PMELs was carried out. Sample consisted of 32 (53.3\%) Indians, 23 (38.3\%) Chinese, 4 (6.7\%) Malays and one (1.7\%) Nepalese. All had histopathological confirmation of their primary existing PMEL as inclusion criteria. A total of 70 primary lesions were detected. The most common PMEL found was oral lichen planus. Of these, $28(46.7 \%)$ patients exhibited bilateral 'mirror-image' lesions $(n=42)$ either synchronously $(n=32 / 42)$ or metachronously $(n=10 / 42)$. The remaining $32(53.3 \%)$ patients had normal-looking contralateral mucosa. Present findings suggest that patients presenting with oral PMELs are at greater risk of developing a second lesion, most probably in the contralateral 'mirror-image' site.

\begin{tabular}{|c|l|}
\hline Author & $\begin{array}{l}\bullet \text { Siar, C. H. } \\
\text { Mah, M. C. }\end{array}$ \\
& $\begin{array}{l}\text { - Gill, P. P. } \\
\end{array}$ \\
\hline Source & European Archives of Oto-Rhino-Laryngology \\
\hline ISSN & $0937-4477$ \\
\hline DOI & $10.1007 /$ s00405-011-1712-x \\
\hline Volume (Issue) & $269(3)$ \\
\hline Page & $999-1004$ \\
\hline Year & 2012 \\
\hline
\end{tabular}

Keyword:

Oral potentially malignant epithelial lesions, Oral mucosa,Clinical,study Screening,lichen-planus,cancer,surveillance,carcinoma, prognosis Criteria,malaysia 
Please Cite As:

URL:

- http://apps.webofknowledge.com search via Accession No >> 000300325300041

- http://www.scopus.com/inward/record.url?eid=2-s2.084857065061\&partnerlD=40\&md5=2d501753cf76c20b63e7ad214eff8eda

- http://www.ncbi.nlm.nih.gov/pubmed/21789676 